\title{
Expression of cofilin 1 is positively correlated with the differentiation of human epithelial ovarian cancer
}

\author{
JIANWEI ZHOU $^{1}$, YINFEN WANG $^{1,2}$, JING FEI $^{1}$ and WEIJIANG ZHANG ${ }^{1}$ \\ ${ }^{1}$ Department of Gynecology, The Second Affiliated Hospital, School of Medicine, Zhejiang University, Hangzhou, \\ Zhejiang 310009; ${ }^{2}$ Department of Gynecology, Ningbo Women and Children's Hospital, Ningbo, Zhejiang 315012, P.R. China
}

Received May 16, 2012; Accepted August 15, 2012

DOI: $10.3892 / \mathrm{ol} .2012 .897$

\begin{abstract}
The aim of this study was to examine the correlation between cofilin 1 expression and differentiation of epithelial ovarian cancer in patients. We immunohistochemically analyzed 30 patients with primary ovarian epithelial carcinomas, 14 patients with borderline epithelial ovarian tumors, 13 patients with benign epithelial ovarian tumors and 10 normal ovarian tissues. All ovarian cancer patients received the standard therapy, including staging laparotomy and adjuvant chemotherapy consisting of carboplatin and paclitaxel. Cofilin 1 expression gradually increased in normal ovarian tissues, benign tumors, borderline tumors and carcinomas, respectively, and there were significant differences among them $(r=0.94, \mathrm{P}<0.05)$. This suggests a positive correlation between the expression of cofilin 1 and tumor differentiation $(\mathrm{r}=0.97, \mathrm{P}<0.05)$. The expression of cofilin 1 may predict the development of ovarian cancer and may be involved in the progression of patients with ovarian carcinoma.
\end{abstract}

\section{Introduction}

Epithelial ovarian cancer is the leading cause of mortality from gynecological cancers, and the majority of patients present with advanced stage $(1,2)$. The 5-year survival rates for stage IIIC and IV patients are 29 and 13\%, respectively $(3,4)$. Researchers have aimed to improve the survival rates of epithelial ovarian cancer patients by studying the effects of early diagnosis, cytoreductive surgery and chemotherapeutic agents (5-8). Recent progress in understanding the pathogenesis of epithelial ovarian cancer suggests that the development and differentiation of ovarian carcinoma is affected by certain

Correspondence to: Dr Weijiang Zhang, Department of Gynecology, The Second Affiliated Hospital, School of Medicine, Zhejiang University, 9 Jiefang Road, Hangzhou, Zhejiang 310009, P.R. China

E-mail: zhou_jianwei@126.com

Key words: cofilin 1, primary ovarian carcinoma, differentiation factors that induced the spread of tumor cells from primary to distal sites $(9,10)$.

A recent study demonstrated that cofilin 1 is a small ubiquitous protein (approximately $19 \mathrm{kD}$ ) that is able to bind monomeric (G) and filamentous (F) actin (11). By severing actin filaments, cofilin 1 increases the number of filament ends for polymerization and depolymerization (12). Cofilin 1 then promotes cytoskeletal dynamics by depolymerizing actin filaments, which is critical for several processes, including cytokinesis and cell motility (13). The activity status of cofilin 1 is directly associated with invasion, intravasation, and metastasis of mammary tumors (14). However, there have been no studies demonstrating a correlation between cofilin 1 expression and the progression and differentiation of patients with ovarian cancer. The aim of the study was to investigate the correlation between cofilin 1 expression and the differentiation of epithelial ovarian cancer in patients, and provide an experimental foundation for the treatment of ovarian cancer.

\section{Materials and methods}

Clinical tissue samples. All clinical tissue samples were obtained from patients at The Women's Hospital of Zhejiang University School of Medicine (Hangzhou, China). The tissues, including 30 primary epithelial ovarian carcinoma (10 well-differentiated cases, 13 moderately differentiated cases and 7 poorly differentiated cases), 14 borderline epithelial ovarian tumors, 13 benign epithelial ovarian tumors and 10 normal ovarian tissues, were collected from patients who underwent surgery. Written/verbal consent was obtained from the patient or the patient's parent/carer, and the use of tissue was approved by the Institutional Review Board of the School of Medicine (Zhejiang University, Zhejiang, China; No. 201104120).

Histological staining and immunohistochemistry. Tissues were preserved in $4 \%$ paraformaldehyde for $24 \mathrm{~h}$. Histological staining and immunohistochemistry were conducted on $8 \mu \mathrm{m}$ sections of paraffin-embedded tissues. All sections were deparaffinized and rehydrated. Sections for histological analysis were stained with $0.3 \%$ cresyl violet (VWR International, Buffalo Grove, IL, USA). Other sections for immunohistochemistry were treated with antigen retrieval containing $10.2 \mathrm{mmol} / \mathrm{l}$ sodium citrate buffer ( $\mathrm{pH}$ 6.1), for 
$20 \mathrm{~min}$ at $95^{\circ} \mathrm{C}$. The sections were then washed in $0.01 \mathrm{M}$ PBS containing $0.3 \%$ Triton X-100 (pH 7.4; PBS-T), immersed in $2 \%$ normal goat serum in PBS for $2 \mathrm{~h}$ at $37^{\circ} \mathrm{C}$, and incubated overnight at $4^{\circ} \mathrm{C}$ with a polyclone cofilin 1 antibody $(1: 100$; Bioss Inc., Woburn, MA, USA) in PBS containing $1 \%$ bovine serum albumin. Once the sections were washed in PBS ( $3 \times 5 \mathrm{~min})$, they were incubated in biotinylated goat-anti-rabbit IgG (1:200; Boster Biological Technology, Ltd., Wuhan, China) in PBS for $4 \mathrm{~h}$ at room temperature, washed in PBS ( $3 \times 5 \mathrm{~min})$, incubated in avidin-biotin-peroxidase complex solution (ABC; 1:100; Boster Biological Technology, Ltd.) for $2 \mathrm{~h}$ at room temperature, then rinsed again in PBS ( $3 \times 5 \mathrm{~min})$. Immunolabeling was visualized using $0.05 \%$ diaminobenzidine (DAB) plus $0.3 \% \mathrm{H}_{2} \mathrm{O}_{2}$ in PBS. After staining, the sections were counterstained using hematoxylin, then dehydrated through ethanol and xylene, and Permount was applied to the coverslips. Rat IgG (1:200; Biomeda Corporation, Foster City, CA, USA) was used instead of a primary antibody as a negative control. Relative cofilin 1 protein expression was determined as the product of the immunostaining intensity and the percent of cells stained.

Image and statistical analysis. Immunostaining intensity was scored as follows: 0 , no staining; 1 , light staining; 2 , moderate staining; and 3, heavy staining. The percent of cell staining was measured as follows: 0 , no detectable staining; 1, 1-33\%; $2,34-66 \%$; and 3,67-100\%. The final staining score was the product of the immunostaining intensity score multiplied by the percent of cells stained score, allowing for a maximal score of 9 and a minimal score of 0 (15). The mean \pm SD for all data were calculated. Statistical analysis was performed using SPSS version 14.0 statistical software (SPSS Inc., St. Louis, MO, USA). The correlation between all the groups was evaluated using the Pearson's correlation coefficient. $\mathrm{P}<0.05$ was considered to indicate a statistically significant difference.

\section{Results}

Diffuse cytoplasmic staining for cofilin 1 with moderate intensity was observed in various proportions of tumor cells. Cofilin 1 was scored for the cytoplasmic immunostaining intensity and the percent of cells stained.

Among the 10 normal ovarian tissues, 5 patients (50\%) did not express cofilin 1 (score $=0$ ), and 5 patients $(50 \%)$ expressed cofilin 1 in $1-33 \%$ of cells (score $=1)$. In the 13 benign epithelial ovarian tumor patients, 8 (61.5\%), $4(30.8 \%)$ and $1(7.7 \%)$ patients expressed cofilin 1 in 1-33\%, 34-66\% and 67-100\% of cells, respectively. Among the 14 borderline epithelial ovarian tumor patients, $1-33 \%$ of cells expressed cofilin 1 in 8 patients $(57.1 \%)$ and $34-66 \%$ in 6 patients $(42.9 \%)$. In the 30 primary epithelial ovarian carcinoma patients, no patients expressed $1-33 \%$ cell staining of cofilin 1 , but 21 patients (70.0\%) expressed 34-66\% and 9 patients (30.0\%) expressed $67-100 \%$ cell staining. The staining score of cofilin 1 gradually increased from normal ovarian to benign tumor, borderline tumor and ovarian carcinoma tissues, respectively $(r=0.94$, $\mathrm{P}<0.05$; Fig. 1 and Table I).

The cofilin 1 expression was also associated with tumor differentiation. The results of a multivariate logistic regression analysis suggested that cofilin 1 was significantly associated
Table I. Immunohistochemistry staining scores for cofilin 1 in normal ovarian, benign ovarian tumor, bordline ovarian tumor and ovarian carcinoma tissues $(\mathrm{r}=0.94, \mathrm{P}<0.05)$.

\begin{tabular}{lcc}
\hline Clinical cases & No. of cases & Staining scores \\
\hline Normal ovarian & 10 & $0.5 \pm 0.5$ \\
Benign ovarian tumour & 13 & $1.5 \pm 0.7$ \\
Bordline ovarian tumor & 14 & $2.5 \pm 0.9$ \\
Ovarian carcinoma & 30 & $6.0 \pm 1.9$ \\
\hline
\end{tabular}

Table II. Immunohistochemistry staining scores for cofilin 1 in ovarian carcinoma tissues with tumor differentiation $(\mathrm{r}=0.97$, $\mathrm{P}<0.05)$.

\begin{tabular}{lcc}
\hline Clinical stage & No. of cases & Staining scores \\
\hline Well-differentiated & 10 & $4.6 \pm 1.0$ \\
Moderately differentiated & 13 & $5.8 \pm 1.7$ \\
Poorly differentiated & 7 & $8.6 \pm 1.1$ \\
\hline
\end{tabular}

with the differentiation grade. Patients with poorly differentiated ovarian carcinoma were more likely to express cofilin 1 compared to those who were moderately and well-differentiated. A total of 34-66\% of cells expressed cofilin 1 in 7 well-differentiated tumor patients (70\% of 10 patients), $67-100 \%$ in 3 well-differentiated tumor patients $(30.0 \%$ of 10 patients), $34-66 \%$ in 7 moderately differentiated tumor patients (53.8\% of 13 patients), $67-100 \%$ in 6 moderately differentiated tumor patients (46.2\% of 13 patients) and $67-100 \%$ in 7 poorly differentiated ovarian carcinoma patients $(100.0 \%$ of 7 patients). The staining score of cofilin 1 gradually increased in well-, moderately and poorly differentiated ovarian carcinoma, respectively ( $r=0.97, \mathrm{P}<0.05$; Fig. 2 and Table II).

\section{Discussion}

Cell migration is the result of a multi-step process initiated by the formation of membrane protrusions in response to migratory and chemotactic stimuli (16). The driving force for membrane protrusion is the localized polymerization of submembrane actin filaments (12). Recently, several studies have revealed that the actin cytoskeleton and its regulatory proteins are dynamically remodeled and are the driving force for cell migration. Cofilin 1 is specifically targeted to filopodia upon stalling of protrusion and during their retraction. Subsequent electron tomography identified that the filopodial actin filament and/or bundle fragmentation may precisely correlate with cofilin 1 accumulation (17). Inhibition of cofilin 1 activity in carcinoma cells with small interfering RNA inhibits cell motility and the invasiveness of carcinoma cells by reducing the assembly and stability of invadopodia $(13,18)$. The overexpression of cofilin enhances the motility of glioblastoma tumor cells in a concentration-dependent fashion, which is likely to contribute to their invasiveness (19). The present study demonstrates that cofilin 1 expression in borderline ovarian tumor tissues was 
A

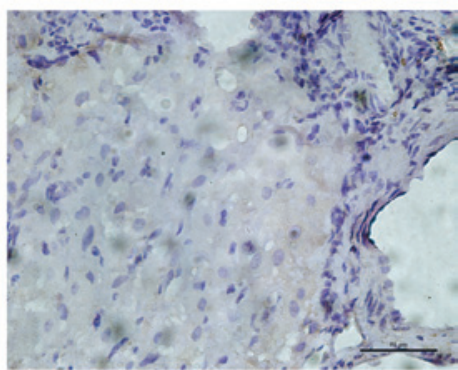

B

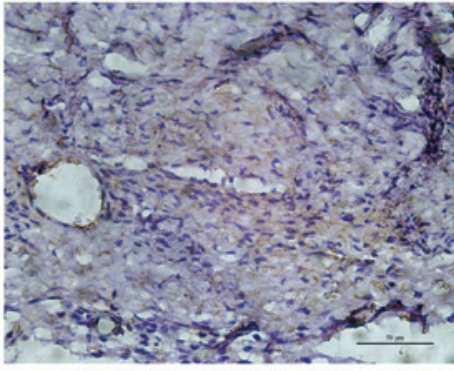

C

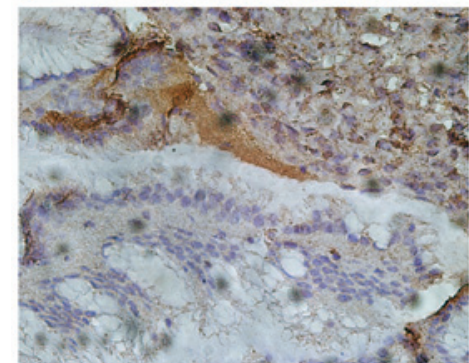

D

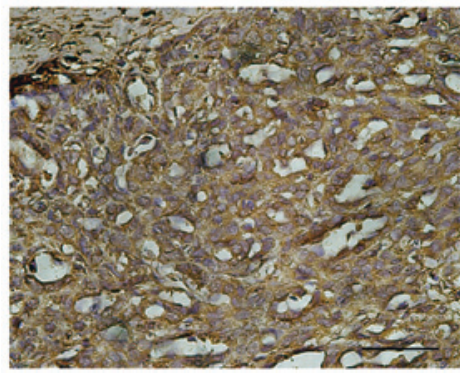

Figure 1. Cofilin 1 immunohistochemical DAB staining in 4 various ovarian tissue types. (A) Normal ovarian tissue; (B) benign ovarian tumor tissue; (C) ovarian bordline tumor tissue; and (D) ovarian carcinoma tissue. DAB, diaminobenzidine.

slightly greater compared with that of benign ovarian tissue, and the expression of cofilin 1 in primary ovarian carcinoma tissues was significantly greater compared with that of the borderline ovarian tumor tissues. The results suggest that cofilin 1 may enhance cell motility and metastasis, and may cause poor prognosis.

As the leading cause of mortality from gynecologic cancers, ovarian cancer cells migrate rapidly and eventually spread throughout the whole peritoneal cavity. The present study revealed that a diffuse cytoplasmic staining for cofilin 1 with moderate intensity was observed in various proportions of tumor cells. No cells expressed cofilin 1 in 7 normal ovarian tissues and only $1-33 \%$ of cells expressed cofilin 1 in 3 normal ovarian tissues. The staining score of cofilin 1 gradually increased from normal ovarian to benign ovarian, ovarian borderline tumor and ovarian carcinoma tissues, respectively.
A

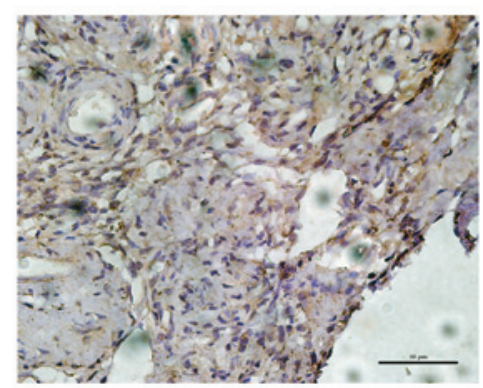

B

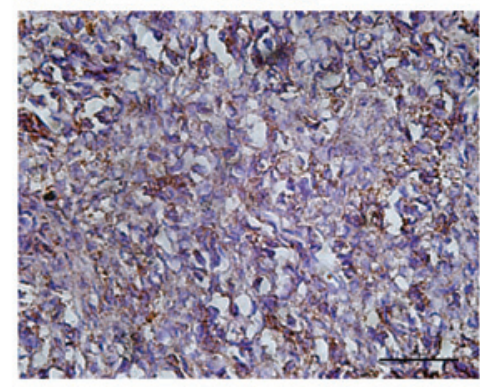

C

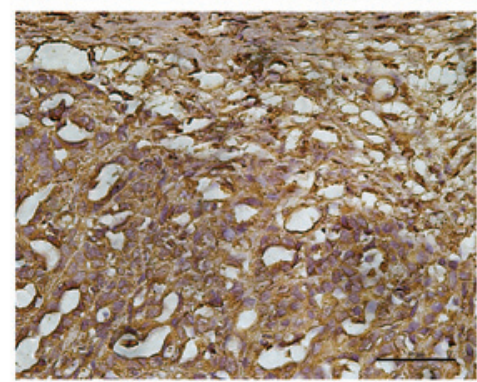

Figure 2. Cofilin 1 immunohistochemical DAB staining in ovarian carcinoma tissues with tumor differentiation. (A) Well-differentiation. (B) Moderately differentiated. (C) Poorly differentiated. DAB, diaminobenzidine.

The invasiveness of malignant cancer cells depends on the altered regulation of cell migration. However, we identified the differential expression of cofilin 1 in tumor differentiation. The data revealed that with the differentiation of ovarian cancers, the level of cofilin 1 expression increased. Meanwhile, the expression of cofilin 1 was positively correlated with the progress of human ovarian cancer differentiation. The present study suggests that the activation of cofilin 1 may promote the proliferation and invasion of cancer cells, leading the development of ovarian cancer.

Our results demonstrate that increased cofilin 1 expression may result in an increase in the progression of ovarian cancer, and indicate that targeting the activities in cancer cells is sufficient to significantly inhibit tumor cell invasiveness. This study provides an experimental foundation for therapeutic strategy, and suggests that RNA Interference Technology or other relevant methods may be used to decrease the level of cofilin 1 and lead to the inhibition of ovarian cancer cell invasion. However, further studies of cofilin 1 in the clinical outcomes of ovarian cancer for are required to clarify these mechanisms.

\section{Acknowledgements}

The study was supported by the Science and Technology Department of Zhejiang province (Zhejiang, China; Grant No. 2011C23093) and the Zhejiang Education Bureau (Zhejiang, China; Grant No. Y201225312). 


\section{References}

1. Yang L, Klint A, Lambe M, et al: Predictors of ovarian cancer survival: a population-based prospective study in Sweden. Int J Cancer 123: 672-679, 2008.

2. Kim PS, Djazayeri S and Zeineldin R: Novel nanotechnology approaches to diagnosis and therapy of ovarian cancer. Gynecol Oncol 120: 393-403, 2011.

3. Chen SS, Michael A and Butler-Manuel SA: Advances in the treatment of ovarian cancer: a potential role of antiinflammatory phytochemicals. Discov Med 13: 7-17, 2012.

4. Chi DS, Eisenhauer EL, Zivanovic O, et al: Improved progression-free and overall survival in advanced ovarian cancer as a result of a change in surgical paradigm. Gynecol Oncol 114: 26-31, 2009.

5. Lutz AM, Willmann JK, Drescher CW, et al: Early diagnosis of ovarian carcinoma: is a solution in sight? Radiology 259: 329-345, 2011

6. Balbi G, Monteverde A, Landino I, Manganaro MA and Franzese C: Cytoreductive surgery and ovarian carcinoma. Acta Biomed 80: 230-233, 2009.

7. Monk BJ and Coleman RL: Changing the paradigm in the treatment of platinum-sensitive recurrent ovarian cancer: from platinum doublets to nonplatinum doublets and adding antiangiogenesis compounds. Int J Gynecol Cancer 19 (Suppl 2): S63-S67, 2009.

8. Li Q, Zhu J, Sun F, Liu L, Liu X and Yue Y: Oncostatin M promotes proliferation of ovarian cancer cells through signal transducer and activator of transcription 3. Int J Mol Med 28: 101-108, 2011.

9. Lutz V, Reuning U, Kruger A, et al: High level synthesis of recombinant soluble urokinase receptor (CD87) by ovarian cancer cells reduces intraperitoneal tumor growth and spread in nude mice. Biol Chem 382: 789-798, 2001.
10. Haller H, Mamula O, Krasevic M, et al: Frequency and distribution of lymph node metastases in epithelial ovarian cancer. Int J Gynecol Cancer 21: 245-250, 2011.

11. Ono S: Mechanism of depolymerization and severing of actin filaments and its significance in cytoskeletal dynamics. Int Rev Cytol 258: 1-82, 2007.

12. Yamaguchi $\mathrm{H}$ and Condeelis $\mathrm{J}$ : Regulation of the actin cytoskeleton in cancer cell migration and invasion. Biochim Biophys Acta 1773: 642-652, 2007

13. Hotulainen P, Paunola E, Vartiainen MK and Lappalainen P Actin-depolymerizing factor and cofilin-1 play overlapping roles in promoting rapid $\mathrm{F}$-actin depolymerization in mammalian nonmuscle cells. Mol Biol Cell 16: 649-664, 2005.

14. Wang W, Mouneimne G, Sidani M, et al: The activity status of cofilin is directly related to invasion, intravasation, and metastasis of mammary tumors. J Cell Biol 173: 395-404, 2006.

15. Shackelford RE, Bui MM, Coppola D and Hakam A: Over-expression of nicotinamide phosphoribosyltransferase in ovarian cancers. Int J Clin Exp Pathol 3: 522-527, 2010.

16. Tsang CM, Lau EP, Di K, et al: Berberine inhibits Rho GTPases and cell migration at low doses but induces $\mathrm{G} 2$ arrest and apoptosis at high doses in human cancer cells. Int J Mol Med 24: 131-138, 2009.

17. Breitsprecher D, Koestler SA, Chizhov I, et al: Cofilin cooperates with fascin to disassemble filopodial actin filaments. J Cell Sci 124: 3305-3318, 2011

18. Yamaguchi H, Lorenz M, Kempiak S, et al: Molecular mechanisms of invadopodium formation: the role of the N-WASP-Arp2/3 complex pathway and cofilin. J Cell Biol 168: 441-452, 2005.

19. Yap CT, Simpson TI, Pratt T, Price DJ and Maciver SK: The motility of glioblastoma tumour cells is modulated by intracellular cofilin expression in a concentration-dependent manner. Cell Motil Cytoskeleton 60: 153-165, 2005. 